\title{
Ponatinib Hydrochloride
}

National Cancer Institute

\section{Source}

National Cancer Institute. Ponatinib Hydrochloride. NCI Thesaurus. Code C78194.

The hydrochloride salt form of an orally bioavailable multitarg eted receptor tyrosine kinase (RTK) inhibitor with potential antiang iog enic and antineoplastic activities. Ponatinib inhibits unmutated and all mutated forms of Bcr-Abl, including T315I, the highly drug therapy-resistant missense mutation of Bcr-Abl. This agent also inhibits other tyrosine kinases including those associated with vascular endothelial growth factor receptors (VEGFRs) and fibroblast growth factor receptors (FGFRs); in addition, it inhibits the tyrosine kinase receptor TIE2 and FMS-related tyrosine kinase receptor-3 (Flt3). RT K inhibition by ponatinib may result in the inhibition of cellular proliferation and angiogenesis and may induce cell death. Bcr-Abl is a fusion tyrosine kinase encoded by the Philadelphia chromosome. 